(2) Open Access Full Text Article

CASE SERIES

\title{
Aqueous misdirection following pars plana vitrectomy and silicone oil injection
}

\author{
This article was published in the following Dove Press journal: \\ Clinical Ophthalmology \\ 20 May 2015 \\ Number of times this article has been viewed
}

\author{
Hammouda H Ghoraba ${ }^{1,2}$ \\ Ali Ahmed Ghali ${ }^{3}$ \\ Hosam Othman Mansour ${ }^{2,3}$ \\ 'Tanta University, Tanta, Egypt: \\ ${ }^{2}$ Magrabi Eye Hospital, Cairo, Egypt; \\ ${ }^{3} \mathrm{Al}-\mathrm{Azhar}$ University, Cairo, Egypt
}

Purpose: To report a retrospective series of seven phakic eyes of seven patients suffering from a malignant glaucoma-like syndrome following pars plana vitrectomy and silicone oil (SO) injection.

Materials and methods: Seven eyes with retinal detachment treated with pars plana vitrectomy with or without scleral buckling with SO tamponade. This was followed by cataract extraction to manage the elevated intraocular pressure (IOP).

Results: This was a retrospective review of seven cases that received pars plana vitrectomy and SO with or without scleral buckling for different causes of retinal detachment (three were rhegmatogenous and four were tractional). After a period ranging from 1 week to 1 month, they presented with malignant glaucoma-like manifestations; high IOP, shallow axial anterior chamber, and remarkable decrease of visual acuity. Atropine eye drops and anti-glaucoma medical treatment (topical and systemic) had been tried but failed to improve the condition. Dramatic decrease of IOP and deepening of the axial anterior chamber was observed in all cases in the first postoperative day after phacoemulsification and posterior chamber foldable intraocular lens implantation with posterior capsulotomy.

Conclusion: Aqueous misdirection syndrome may be observed following pars plana vitrectomy and SO tamponade. This must be differentiated from other causes of post vitrectomy glaucoma. Cataract extraction with posterior capsulotomy controls the condition.

Keywords: malignant glaucoma, pars plana vitrectomy, silicone oil

\section{Introduction}

Aqueous misdirection syndrome may be observed after pars plana vitrectomy (PPV) and silicone oil (SO) injection. Cataract extraction is a curative surgery for this condition as well as for other causes of aqueous misdirection.

Uncomplicated PPV may be associated with elevated intraocular pressure (IOP) due to either open or closed angle mechanisms. In one study, $43.3 \%$ of patients undergoing PPV had IOP above $30 \mathrm{mmHg}$ in the acute postoperative period. ${ }^{1}$ Some authors suggested that there is a significant number of patients with acute elevations in IOP in the first 24 hours after surgery followed by normalization of the pressure curve in many of them. ${ }^{2}$ Risk factors for IOP elevation demonstrated in that study include intraoperative or previous scleral buckling, intraoperative lensectomy or endophotocoagulation, and postoperative fibrin formation. A pre-existing history of glaucoma did not increase the rate of IOP elevation after PPV. ${ }^{1}$ A variable incidence of postoperative IOP elevation following SO injection has been reported. A recent study found a lower incidence (11\%) of glaucoma after PPV with injection of highly purified SO (5,000 centistokes). ${ }^{3}$ Topical and systemic anti-glaucoma medications achieve effective control of IOP in the majority of patients, and topical steroids are often administered after surgery to
Correspondence: Hosam Othman Mansour

Department of Ophthalmology, Al-Azhar University, Autostrad st, Nasr City,

Cairo 020, Egypt

Email drhmansor@yahoo.com 
control inflammation. Guarded filtration surgery is generally not recommended due to upward migration of SO as well as conjunctival scarring following PPV. However, a glaucoma drainage implant is useful for the treatment of elevated IOP in eyes that do not respond to medical therapy. ${ }^{3,4}$ In patients with increased IOP after SO injection, removal of SO may not be enough to solve the underlying problem. ${ }^{5}$

Aqueous misdirection syndrome has been observed following many types of intraocular surgery, including cataract surgery with or without intraocular lens (IOL) implantation, ${ }^{6}$ trabeculectomy, ${ }^{7}$ and Seton implant surgery. ${ }^{8}$ It has been reported following laser surgical procedures such as laser suture lysis of trabeculectomy flap sutures, ${ }^{9}$ laser iridotomy, ${ }^{10} \mathrm{Nd}$ :YAG laser posterior capsulotomy, ${ }^{11}$ and noncontact Nd:YAG laser photocoagulation. ${ }^{12}$ Massicotte and Schuman have reported two cases of malignant glaucomalike syndrome following PPV and 15\% perfluoropropane (C3F8) gas injection, despite the fact that vitrectomy has traditionally been considered a curative treatment of aqueous misdirection. ${ }^{13}$

\section{Materials and methods}

This was a retrospective series of seven phakic eyes with retinal detachment of different etiologies treated by PPV and SO. All surgeries were done by the same surgeon (HHG). Three port PPV combined with 360 degree encircling scleral band was done in two patients. The other five patients had no scleral band. The band was fixed in the four quadrants with one polyester suture and in the upper nasal quadrant with a sleeve. Vitreous surgery consisted of core vitrectomy; triamcinolone assisted posterior vitreous detachment. Tractional membranes were dissected using curved vitreous scissors until the retinal surface came free. Perfluorocarbon liquid was used to flatten the retina in rhegmatogenous detachments. Vitrectomy was completed as safe as possible. Air fluid exchange and drainage of sub-retinal fluid was done. Endo laser was applied around breaks; pan retinal photocoagulation was completed in tractional cases and as 360 degree barrage in rhegmatogenous cases. SO 5,000 centistokes was finally injected. After failure of medical treatment to control the elevated IOP phacoemulsification with IOL implantation and posterior capsulotomy was done for all cases.

\section{Postoperative care}

All the included patients had slit lamp examination in the first postoperative day to detect any early postoperative complications such as IOP rise, fundus examination and color fundus photography were done. The follow-up was scheduled to be at 1 week, 3 weeks, 6 weeks, and then every 8 weeks. At each visit the following data were recorded, best corrected visual acuity, IOP, SO in the anterior chamber, and retinal attachment.

\section{Results}

The medical records of seven phakic eyes with retinal detachment of different etiologies treated by PPV and SO were retrospectively reviewed, four were males and three were females. Age range was 30-55 years. Four cases had tractional and three cases had rhegmatogenous retinal detachment. All of them received PPV and SO (5,000 centistokes) tamponade. All seven cases presented with increases IOP (ranging from 26 to $50 \mathrm{mmHg}$ ) at different times, two cases by the 1 st week, two cases by the 2 nd week, one patient by the 3 rd week and two patients by the 4th week. Other clinical signs were shallow axial anterior chamber, forward lens displacement, and deterioration of vision in all cases. Medical treatment of glaucoma (systemic and topical) was tried for 24-48 hours, but failed to control the IOP. A diagnosis of aqueous misdirection was made and phacoemulsification was done after which a dramatic decrease of the IOP was observed on the first postoperative day, associated with deepening of the axial anterior chamber. No SO was observed in the anterior chamber in any of the seven cases. In all cases no rebound increase of the IOP was observed throughout the follow-up time. The final IOP ranged from $10-16 \mathrm{mmHg}$. The final visual acuity was related to the retinal status. Full data of patients before and after phacoemulsification is shown in Tables 1 and 2.

\section{Discussion}

Malignant glaucoma or aqueous misdirection syndrome is a rare secondary angle-closure glaucoma characterized by axial shallowing of the anterior chamber, elevated IOP, and the absence of suprachoroidal fluid or blood. ${ }^{14}$ Approximately half of patients with malignant glaucoma respond to medical therapy with mydriatics, cycloplegics, aqueous suppressants, and dehydrating agents within 4-5 days. ${ }^{15}$ The success of laser treatment for malignant glaucoma depends on the disruption of the anterior hyaloid face and creation of communication between the vitreous and the anterior chamber. ${ }^{14}$ PPV is reserved for those patients with refractory malignant glaucoma. One would not expect aqueous misdirection to follow PPV performed for other indications. ${ }^{13}$

In our series, we reported aqueous misdirection in seven patients who underwent PPV, three of them had rhegmatogenous retinal detachment and four had diabetic tractional 
Table I Patient characteristics and clinical data before pars plana vitrectomy

\begin{tabular}{lllllll}
\hline Case number & Age (years) & Sex & Laterality & Diagnosis & IOP $(\mathbf{m m H g})$ & Procedure \\
\hline I & 47 & M & OD & VH + TRD & I4 & PPV + SO \\
2 & 55 & M & OD & Rhg RD & 10 & SB + PPV + SO \\
3 & 30 & M & OD & Rhg RD & 12 & SB + PPV + SO \\
4 & 33 & F & OD & TRD & 10 & PPV + SO \\
5 & 53 & M & OD & Rhg RD & 13 & PPV + SO \\
6 & 62 & F & OS & TRD & 12 & PPV + SO \\
7 & 54 & F & OD & VH + TRD & 14 & PPV + SO \\
\hline
\end{tabular}

Abbreviations: M, male; F, female; OD, right eye; OS, left eye; SO, silicone oil; VH, vitreous hemorrhage; TRD, tractional retinal detachment; Rhg, rhegmatogenous; RD, retinal detachment; IOP, intraocular pressure; PPV, pars plana vitrectomy; SB, scleral buckling.

retinal detachment. SO (5,000 centistokes) was injected in all cases. Scleral buckling was combined with PPV in two cases of rhegmatogenous detachment. Massicotte and Schuman have reported two cases of malignant glaucomalike syndrome following PPV, 15\% perfluoropropane (C3F8) gas injection was used. They explained this paradox may be due to surgical technique, particularly, a partial vitrectomy leaving the anterior hyaloid intact. ${ }^{13}$ Byrnes et al ${ }^{16}$ stated that, disruption of the anterior hyaloid without damaging the crystalline lens during PPV is a technical challenge, particularly, in phakic patients. ${ }^{16}$

Harbour et $\mathrm{al}^{15}$ reported a 29\% (two of seven) recurrence of malignant glaucoma in phakic patients following PPV. Byrnes et $\mathrm{al}^{16}$ reported persistent or recurrent ciliary block glaucoma in 50\% (five of ten) of phakic patients and in $10 \%$ (one of ten) of pseudophakic eyes in their series of patients who underwent vitrectomy for malignant glaucoma. In our series, a dramatic decrease of IOP was observed following phacoemulsification and foldable posterior chamber (PC) IOL implantation and posterior capsulotomy, and associated with deepening of the axial anterior chamber. No recurrence of aqueous misdirection was reported in this series throughout the follow-up time (from 6 months to 2 years). Momoeda et al reported no recurrence of malignant glaucoma after vitrectomy combined with lensectomy in their five patients. ${ }^{17}$ It is possible that persistent anterior hyaloid material is responsible for the recurrence of malignant glaucoma, ${ }^{16}$ and new occurrence of malignant glaucoma according to previous reports. ${ }^{13}$ A case of recalcitrant malignant glaucoma following PPV, scleral buckle, and extracapsular cataract extraction with PC IOL implantation has been reported. ${ }^{18}$ The aqueous misdirection recurred despite multiple interventions, including medical treatment, transiently successful Nd:YAG laser capsule-hyaloidectomy, and surgical disruption of the anterior hyaloid face. It finally resolved after repeated PPV with hyaloido-capsulo-iridectomy. There had been no recurrence 7 months after this procedure. The authors concluded that the surgical treatment of aqueous misdirection refractory to more conservative measures should include adequate disruption of the anterior hyaloid to prevent recurrence. ${ }^{18}$

Some authors reported doubt about the role of scleral buckling or endolaser in the pathogenesis in this syndrome. ${ }^{13}$

In our series scleral buckling was done in two of seven cases, and this may rule out the hypothesis of relative SO overfill due to choroidal congestion (edema) and the decreases size of the globe. The coexistence of such condition with scleral buckling may explain the unclear mechanism of this syndrome.

Although PPV was considered curable treatment for aqueous misdirection the condition may be observed after PPV and SO injection. The condition should be considered in the differential diagnosis of post vitrectomy glaucoma.

Table 2 Postoperative data

\begin{tabular}{|c|c|c|c|c|}
\hline Case number & $\begin{array}{l}\text { Timing of presentation } \\
\text { of IOP rise (after PPV) }\end{array}$ & $\begin{array}{l}\text { IOP }(\mathrm{mmHg}) \\
\text { before phaco }\end{array}$ & $\begin{array}{l}\text { Axial anterior } \\
\text { chamber }\end{array}$ & $\begin{array}{l}\text { Final IOP }(\mathrm{mmHg}) \\
\text { after phaco }\end{array}$ \\
\hline I & Ist W & 50 & Shallow & 12 \\
\hline 2 & Ist M & 26 & Shallow & 14 \\
\hline 3 & 2nd W & 40 & Shallow & II \\
\hline 4 & $3 r d W$ & 50 & Shallow & 16 \\
\hline 5 & Ist M & 48 & Shallow & 13 \\
\hline 6 & Ist W & 38 & Shallow & 12 \\
\hline 7 & 2nd W & 50 & Shallow & 10 \\
\hline
\end{tabular}

Abbreviations: W, week; M, month; PPV, pars plana vitrectomy; IOP, intraocular pressure; phaco, phacoemulsification. 
The diagnosis relies mainly on the clinical basis and by exclusion of other causes of secondary glaucoma (SO overfill and papillary block glaucoma).

The precise mechanism of aqueous misdirection following vitrectomy and SO injection remains unclear. We believe that cataract extraction with posterior capsulotomy at the time of phacoemulsification is curative for this condition.

\section{Disclosure}

The authors report no conflicts of interest in this work.

\section{References}

1. Han DP, Lewis H, Lambrou FH, Mieler WF, Hartz A. Mechanisms of intraocular pressure elevation after pars plana vitrectomy. Ophthalmology. 1989;96(9):1357-1362.

2. Desai UR, Alhalel AA, Schiffman RM, Campen TJ, Sundar G, Muhich A. Intraocular pressure elevation after pars plana vitrectomy. Ophthalmology. 1997;104(5):781-785.

3. Al-Jazzaf AM, Netland PA, Charles S. Incidence and management of elevated intraocular pressure after silicon oil injection. $J$ Glaucoma. 2005; 14(1):40-46.

4. Ishida K, Ahmed IK, Netland PA. Ahmed glaucoma valve surgical outcomes in eyes with and without silicon oil endotamponade. J Glaucoma. 2009; 18(4):325-330.

5. Moisseiev j, barak A, Manaim T, Triester G. Removal of silicon oil in the management of glaucoma in eyes with emulsified silicone. Retina. 1993;13(4):290-295.

6. Duy TP, Wollensak J. Ciliary block (malignant) glaucoma following posterior chamber lens implantation. Ophthalmic Surg. 1987; 18(10):741-744.
7. Ellis PP. Malignant glaucoma occurring 16 years after successful filtering surgery. Ann Ophthalmol. 1984;16(2):177-179.

8. Melamed S, Cahane M, Gutman I, Blumenthal M. Postoperative complications after Molteno implant surgery. Am J Ophthalmol. 1991; 111(3):319-322.

9. Scalafani M, Liebmann JM, Ritch R. Malignant glaucoma following argon laser release of sclera flap sutures after trabeculectomy. Am J Ophthalmol. 1989;108(5):597-598.

10. Cashwell LF, Martin TJ. Malignant glaucoma after laser iridotomy. Ophthalmology. 1992;99(5):651-659.

11. Mastropasqua L, Ciancaglini M, Carpineto P, Lobefalo L, Gallenga PE. Aqueous misdirection syndrome: a complication of neodymium: YAG posterior capsulotomy. J Cataract Refract Surg. 1994;20(5): 563-565.

12. Hardten DR, Brown JD. Malignant glaucoma after Nd: YAG cyclophotocoagulation. Am J Ophthalmol. 1991;111(2):245-247.

13. Massicotte EC, Schuman JS. A malignant glaucoma-like syndrome following pars plana vitrectomy. Ophthalmology. 1999;106(7): $1375-1379$.

14. Epstein DL. The malignant Glaucoma syndromes. In: Epstein DL, Allingham RR, Schuman JS, editors. Chandler and Grant's Glaucoma, 4th ed. Baltimore: Williams and Wilkins; 1997:285-303.

15. Harbour JW, Rubsamen PE, Palmberg P. Pars plana vitrectomy in the management of phakic and pseudophakic malignant glaucoma. Arch Opthalmol. 1996;114(9):1073-1078.

16. Byrnes GA, Leen MM, Wong TP, Benson WE. Vitrectomy for ciliary block (malignant) glaucoma. Ophthalmology. 1995;102(9):1308-1311.

17. Momoeda S, Hayashi H, Oshima K. Anterior pars plana vitrectomy for phakic malignant glaucoma. Jpn J Ophthalmol. 1983;27(1):73-79.

18. Zacharia PT, Abboud EB. Recalcitrant malignant glaucoma following pars plana vitrectomy, scleral buckle, and extracapsular cataract extraction with posterior chamber IOL implantation. Ophthalmic Surg Lasers. 1998;29(4):323-327.
Clinical Ophthalmology

\section{Publish your work in this journal}

Clinical Ophthalmology is an international, peer-reviewed journal covering all subspecialties within ophthalmology. Key topics include: Optometry; Visual science; Pharmacology and drug therapy in eye diseases; Basic Sciences; Primary and Secondary eye care; Patient Safety and Quality of Care Improvements. This journal is indexed on Submit your manuscript here: http://www.dovepress.com/clinical-ophthalmology-journal

\section{Dovepress}

PubMed Central and CAS, and is the official journal of The Society of Clinical Ophthalmology (SCO). The manuscript management system is completely online and includes a very quick and fair peer-review system, which is all easy to use. Visit http://www.dovepress.com/ testimonials.php to read real quotes from published authors. 